\title{
Andrea Plöger
}

\section{Die Proteste der Refugees Eine Bewegung von den Rändern ins Zentrum Europas}

In den letzten zwei Jahren ist mit den verschiedenen Refugee-Protesten erstmals eine soziale Bewegung in Deutschland entstanden, die über einzelne Proteste und Kampagnen hinausweist und ein gesamtgesellschaftliches Ziel verfolgt. Viele der AktivistInnen sind aufgrund der Revolutionen und nachfolgenden Konflikte oder Militärinterventionen nach Europa gekommen. Von den Rändern der EU und den Krisenländern haben sie sich auf den Weg ins Zentrum gemacht. Als Hauptleidtragende einer neoliberalen Globalisierungspolitik, des Abbaus noch bestehender Subsistenz- und Wohlfahrtsstaatsstrukturen sowie dem Einsatz von Kontrolle und Zwang ist ihr Schicksal auch exemplarisch für das, was eine neoliberale Umstrukturierung für „die 99 Prozent“ bereithält. Insofern korrespondiert ihr Aufstand gegen Isolation und für Teilhabe mit den Zielen anderer kontemporärer Protestbewegungen. Hier stellt sich die Frage, ob eine Fortführung der Austeritätspolitik weiterhin mit Verweis auf „Einwanderer, die die Sozialsysteme missbrauchen" und auf die „Pleitegriechen“"vermittelt werden kann - auf Kosten der ,Ränder ' und zugunsten der EU-Zentren, gegen die ohnehin schon in die Illegalität Gedrängten, auf Kosten des Gros der Bevölkerung und zum Nutzen von Konzernen und Banken. Oder können die sogenannten Global Justice Movements Spaltungen überwinden, an Einfluss gewinnen und zu ernsthaften Antipoden werden (vgl. Brand 2012) und dabei auch die Kehrseite des Rechts auf Bewegungsfreiheit - das Recht, zu bleiben - und damit die gewaltsame und teilweise kriegerische Zerstörung von Subsistenzstrukturen in den Peripherien und die daraus folgende „Produktion der Migration“ stärker thematisieren?

\section{Die Refugee-Bewegung als Herausforderung für die Migrations- und Bewegungsforschung}

Angesichts der Bedeutung der Refugee-Bewegung als einer zentralen gegenhegemonialen Kraft kann zur Analyse m.E. nicht allein auf die Migrationsoder Bewegungsforschung zurückgegriffen werden. Vielmehr muss sich eine kritische Analyse des gesellschaftlichen und internationalen Stellenwerts der 
nunmehr seit zwei Jahren aktiven Refugee-Bewegung in Deutschland auch auf gesamtgesellschaftliche und globalisierungskritische Theorien stützen, die über die Migrationsforschung hinausgehen, um die Wirkung dieser Bewegung und die Zusammenhänge ihrer Entstehung erfassen zu können. Die kritische Migrationsforschung hat - korrespondierend mit der zunehmenden Präsenz des Themas in der öffentlichen Diskussion - in den letzten zwanzig Jahren verschiedene Richtungen entwickelt und sich stärker auf andere Forschungszweige bezogen. Zudem sind Netzwerke kritischer Migrationsforschung und migrationspolitischer Gruppen wie Kanak Attack, Trans Migration und kritnet entstanden, die den Refugee-Protesten nahe stehen und oftmals ForscherInnen und AktivistInnen in einer Person sind. Kritische ForscherInnen eröffneten vor mehr als zehn Jahren die Debatte um die „Autonomie der Migration“ (vgl. u.a. Moulier Boutang 2002; Trans Migration 2005; Mezzadra 2011; Hardt/Negri 2003) bzw. eine feministische Einschränkung des Konzeptes als „Eigensinnigkeit“ der Migration (Benz/Schwenken 2005). Migration wird hier auch als „social and political movement in the literal sense of the words" verstanden (Papadopoulos et al. 2008: 202). Die Kritik an diesem Konzept kam nicht zuletzt von Geflüchteten und MigrantInnen selbst, die darin eine „Romantisierung“ der Migration sahen (Omwenyeke 2004). Sandro Mezzadra (2011: 1) hat den Ansatz verteidigt und betont, dass das Gewinnbringende des Konzeptes der Blick auf Migration als „tense and conflict-driven process, in which subjective movements and struggles of migrationare an active and fundamental factor" sei. Wichtig erscheint mir ebenfalls an der Debatte - vor allem im Hinblick auf die derzeitigen Kämpfe -, dass die Subjektivität von MigrantInnen und Geflüchteten im Mittelpunkt der Debatte steht und Migration nicht nur als Ergebnis gewaltsamer kapitalistischer Inwertsetzungsprozesse, sondern auch als eine mögliche Störung und widerständige Praxis erscheinen kann.

Ein weiterer Strang kritischer Sozialwissenschaft in der Migrationsforschung nach 1990 sind die Citizenship Studies, die auch in der Refugee-Bewegung diskutiert werden. Die Fokussierung des Begriffs „auf die Frage nach Ein- und Ausschluss" ermögliche es, so Henrik Lebuhn (2013: 236), den „Versuch, MigrantInnen vom Zugang zu öffentlichen Ressourcen auszuschließen, unter anderem als Versuch der Ethnisierung von Verteilungskonflikten zwischen Klassen und innerhalb von Klassen (zu) entschlüsseln“. Inzwischen hat ein Teil der RefugeeBewegung, die sich Non-Citizens nennen, dort angesetzt und eine gleichberechtigte Teilhabe gefordert, die ihnen als Menschen, „die vor der Unterdrückung in einer Gesellschaft geflüchtet sind, in der sie den Wohlstand der ,sicheren Länder mit geschaffen haben, (...) weiterhin verwehrt wird“ (refugee tent action 2012).

Den wissenschaftlichen Hintergrund vieler kritischer Migrationsforschungsansätze und auch der vorliegenden Analyse bildet die Weltsystemtheorie. Sie 
ermöglicht es, Migration und vor allem auch die Schaffung, irregulärer 'Migration als einen zielgerichteten und mit kapitalistischer Entwicklung einhergehenden fortwährenden Prozess zu theoretisieren (vgl. Wallerstein 1984). Geflüchtete und MigrantInnen werden so permanent durch die Zerstörung von Subsistenzökonomien und gesellschaftlicher Gefüge geschaffen. Dies ist vor allem für das Verständnis von dem wichtig, was ich die „Produktion der Migration“ nennen möchte. Der sogenannte Arabische Frühling und die nachfolgenden kriegerischen Konflikte, Militärinterventionen und geostrategischen Verschiebungen produzieren seitdem Geflüchtete und MigrantInnen in hoher Zahl. Im Schatten dieser Kriege und Konflikte sorgen Freihandelsabkommen, das sprunghaft gestiegene Land Grabbing, der in den Freiräumen zusammenbrechender Systeme vorangetriebene Ausbau von Minen und unkontrollierten Giftmülldeponien sowie der Abbau staatlicher Gesundheits- und Bildungssysteme dafür, dass die lokalen, regionalen und nationalen Ökonomien von den Transferzahlungen der MigrantInnen und Geflüchteten abhängig werden. Die sich dadurch zuspitzenden sozialen und politischen Konflikte und die damit einhergehenden autoritären und diktatorischen Regierungsregime tragen wiederum ihrerseits zur „Produktion der Migration“ bzw. zur „Produktion von Flucht und Exil“ bei.

In dem vorliegenden Text ergänze ich Ansätze der Weltsystemtheorie durch Forschungen über den Umbau des fordistischen Staates zum postfordistischen Staat und den Aufbau des Kontroll- und Überwachungsstaates (vgl. Hardt/Negri 2003, 2013; Butterwege 2013), um die Bedeutung der Refugee-Bewegung für die Zielländer von MigrantInnen und Geflüchteten in der EU und insbesondere in Deutschland als neuer EU-Zentralmacht besser nachvollziehen zu können. Die Refugee-Proteste, wie sie seit mehr als zwei Jahren in einigen europäischen Städten und insbesondere auch in Berlin den öffentlichen Raum besetzen, sind m.E. auch im Kontext der „Neoliberalisierung“ der Städte (zu Berlin vgl. Holm 2014; Bouali/Gude 2014) bzw. der „neoliberalisierenden“Stadt (vgl. Mayer 2013a, b) zu betrachten. Obwohl in den letzten Jahren in verschiedenen wissenschaftlichen Disziplinen vermehrt zum Thema Migration publiziert wurde und die Bewegungsforschung sich seit einiger Zeit des Themas verstärkt annimmt, war das Auftauchen und vor allem Bleiben der Refugee-Bewegung in Deutschland seit 2012 eine Überraschung für ForscherInnen und Netzwerke. ForscherInnen und AktivistInnen diagnostizierten noch einige Jahre vorher eine „Schwäche“ migrantischer Proteste (Omwenyeke 2004) und sahen kaum Potenzial zur Bildung einer eigenständigen sozialen Bewegung (vgl. Rucht/Heitmeyer 2008). 


\section{Die Refugee-Proteste formieren sich in Deutschland erstmals zu einer sozialen Bewegung}

Am 29.1.2012 - ein Jahr nach Beginn des Arabischen Frühlings - erhängte sich der iranische Geflüchtete Mohammad Rashep „wegen der Frustration, Angst vor der Abschiebung und der Art und Weise, wie non-citizen in diesem Teil der Welt Deutschland behandelt (werden)" in einem Asylbewerberheim in Würzburg (siehe refugeestruggle.org/de/ueber-uns). Selbstmorde kommen in der Isolation der Asylunterkünfte und unter Androhung von Abschiebung relativ häufig vor. Dennoch ist die Chance für Widerstand gering; die Isolation in Camps, die Perpetuierung einer Flucht- und Migrationssituation aufgrund des Dublin Übereinkommens ${ }^{1}$, eine angegriffene psychische und physische Gesundheit und die vielfältigen Hintergründe der MigrantInnen und Geflüchteten erschweren eine gemeinsame Organisierung oder das Entstehen einer Bewegung. Die Gelegenheitsstrukturen für Protest sind äußerst ungünstig, der Mangel an finanziellen und machtpolitischen Ressourcen und Zugängen eklatant.

Resultat der Isolation und Entrechtung von Asylsuchenden, MigrantInnen und Geflüchteten in Deutschland ist auch ein „mangelnde(r) Organisierungsgrad“von Refugee-Protesten (Josten 2012: 88) und ein Mangel an „sektorübergreifende(n) Allianzen" zwischen Advocacy-Netzwerken (wie etwa die NGO Pro Asyl) und migrantischer Selbstorganisierung (Schwenken 2007: 52). Hinzu kommt, dass für Geflüchtete und MigrantInnen - insbesondere wenn sie isoliert in Lagern untergebracht oder illegalisiert sind - kaum die Möglichkeit besteht, Zugang zu einer weiter gefassten Öffentlichkeit in Deutschland zu erlangen. Die (mediale) Kommunikation ihrer Botschaften an eine breitere Öffentlichkeit, wie sie ressourcenarme soziale Bewegungen (vgl. Neidhardt 1994; Plöger 2014) sonst oftmals nutzen, ist hier weitgehend versperrt. Zehn AsylbewerberInnen treten dennoch Wochen später in einen Hungerstreik, um auf die Bedingungen für Asylsuchende in Deutschland und der EU aufmerksam zu machen. Anfang Juli 2012 beginnen sie die Refugee Tent Action, weil es keine Antworten von offizieller Seite gibt. ${ }^{2}$ Nachdem sich zunächst vor allem AsylbewerberInnen den lokalen Zeltprotesten angeschlossen und den Slogan „Break Isolation“ damit in die Tat umgesetzt haben, ist der nächste Schritt ein Marsch von Würzburg nach Berlin. Im September desselben Jahres übertreten zehn Asylsuchende öffentlich

1 Das Dublin-Übereinkommen trat 1997 für alle EU Länder in Kraft und wurde 2003 und 2013 mit Dublin II und III erweitert und angeglichen. Inhalt der Vereinbarungen ist die Festlegung von Asylsuchenden auf die Länder, über die sie den Schengenraum erreicht haben. Sie werden genötigt, dort ihren Asylantrag zu stellen.

2 Blog der Refugee Tent Action: refugeetentaction.net. 
die Landesgrenze von Bayern zu Thüringen - bis 1989 Grenze zwischen DDR und BRD. Sie zerreißen ihre Aufenthaltsgenehmigungen, die sie aufgrund der in Deutschland geltenden "Residenzpflicht“ zwingen, ihren Landkreis oder Bundesland - je nach Auslegung - nicht zu verlassen. ${ }^{3}$

Am 6. Oktober erreichen etwa 200 Asylsuchende und UnterstützerInnen Berlin und bauen mit Genehmigung des Bezirks ein Protestcamp am Oranienplatz in Kreuzberg auf. Die Demonstration für die drei zentralen Forderungen des Asylstrikes - Abschaffung der Residenzpflicht, Stopp der Abschiebungen und ein Ende der Lagerunterbringung - ist mit ca. 6.000 Teilnehmenden die bis dato größte Demonstration für die Rechte von MigrantInnen und Geflüchteten in Deutschland. ${ }^{4}$ Zur Vorweihnachtszeit treten Geflüchtete öffentlich vor dem Brandenburger Tor in einen Hungerstreik. Inzwischen berichten die Mainstreammedien regelmäßig über das Camp am Oranienplatz und den Hungerstreik. Auch internationale Medien wie AlJazeera informieren jetzt über die Bedingungen für Asylsuchende in Deutschland. ${ }^{5}$ Zur selben Zeit wird die Gerhard-HauptmannSchule in Berlin-Kreuzberg besetzt und dann vom Bezirk als Winterquartier zur Verfügung gestellt. Der „cycle of contention“, die Protestwelle (vgl. Koopmans 2004; Tarrow 1998), wird größer und der Protest breitet sich über verschiedene gesellschaftliche Gruppen aus. Ein sogenanntes UnterstützerInnennetzwerk entsteht. Die Refugee-Proteste können erstmals in Deutschland nach den gängigen Kriterien der Bewegungsforschung als soziale Bewegung definiert werden, da sie inzwischen mehr als „kollektive Proteste“ oder eine „singuläre Kampagne“ sind (vgl. Tilly 2004: 7) und auch versuchen, „Einfluss auf sozialen Wandel zu nehmen" (Roth/Rucht 2008: 13).

Mit der zunehmenden Breite der Proteste und der Formierung einer sozialen Bewegung nehmen aber auch die Spannungen zu. Während sich soziale Bewegungen in der Regel in der Phase der Verbreiterung und des Übergangs von einzelnen Protesten zu einer Bewegung diversifizieren und dadurch die partikularen Interessen und Hintergründe der AktivistInnen offener zutage treten, potenziert

3 Ein besonderer Ansatz der deutschen Asylpolitik ist die Anordnung der sogenannten Residenzpflicht, also die Beschränkung der Bewegungsfreiheit von Asylsuchenden auf den ihnen zugewiesenen Landkreis oder das Bundesland. Sie bedeutet - zusammen mit der Unterbringung in der Peripherie von Städten und Gemeinden, dem Arbeits- und Studienverbot und dem mangelnden Zugang zu Sprachkursen - eine Isolation von Asylsuchenden von der lokalen Bevölkerung. Dies entspricht in vielen Gegenden auch dem Willen der WählerInnen der verantwortlichen Abgeordneten.

4 Blog des Asylstrike:asylstrikeberlin.wordpress.com.

5 Eine Auswahl von Artikeln und links zu Radio-und TV Beiträgen findet sich auf asylstrikeberlin.wordpress.com 
sich die Wirkung der „cross-cutting cleavages“im Fall der Refugee-Bewegung erheblich (vgl. Rucht/Heitmeyer 2008: 586).

Gegen Ende des Jahres schließen sich die ersten Geflüchteten an, die aufgrund der Militärintervention Libyen verlassen mussten. Es kommen auch immer mehr Menschen aus Mali, wo der Tuareg-Aufstand - geführt mit Waffenbeständen der libyschen Armee - von Al Qaida im Maghreb (AQMI) und der Bewegung für Einheit und den Dschihad in Westafrika (MUJAO) überrollt wird. ${ }^{6}$ Viele erreichen Deutschland über Italien, dessen Asylsystem kaum mehr existiert und das als einen der ersten Schritte zur Umsetzung von Austeritätsprogrammen die Leistungen für AsylbewerberInnen - ob anerkannt oder nicht - streicht.

So ändert sich 2013 die Zusammensetzung des Protestbündnisses weiter. Die Geflüchteten, die über Italien eingereist sind und unter das Dublin-Übereinkommen fallen, haben keinen Zugang zu Unterstützung in Deutschland und sind in die Illegalität gezwungen. Sie sind auf Freiräume wie das Camp am Berliner Oranienplatz oder die Gerhard-Hauptmann-Schule sowie auf die Verpflegung durch die Volksküche am Oranienplatz und die Sanitäranlagen und medizinische Versorgung in den noch bestehenden Unterstützungsstrukturen aus der Hausbesetzerbewegung der 1980er Jahre angewiesen. Die „Neoliberalisierung der Städte“, die sich durch die Verfolgung einer ,aggressiven Strategie des Wirtschaftswachstums“, durch ,unternehmerische Formen der Governance“, die Privatisierung öffentlicher Güter und Dienstleistungen und folglich eine soziale Polarisierung (Mayer 2013a) sowie den Umbau des Staates insgesamt auszeichnet, haben dazu geführt, dass viele „UnterstützerInnen“ inzwischen selbst beengt wohnen und in der Prekarität überleben. Auf der anderen Seite haben viele UnterstützerInnen der Vorläuferbewegungen kaum noch Berührungspunkte mit sozialen Bewegungen, da der errungene Arbeitsplatz oder Lebensraum verteidigt und finanziert werden muss. Der Akt der Unterstützung bedeutet insofern schon ein Auflehnen gegen die „neoliberale Gleichgültigkeit“ und ein Festhalten an „einer Idee der Solidarität“ (Arps 2014).

Die Drittstaatenregelung in Form von Dublin I bis III verdeutlicht den Anspruch einiger Staaten, offiziell als „Kern-Europa“ aufzutreten, und führt zu einem Mangel an Protestadressen, was die Formierung der Bewegung zusätzlich erschwert. Zwar sind die Refugee-Proteste von Beginn an in einen transnationalen Kontext eingebettet, aber der Mangel an Bewegungsfreiheit hindert AktivistInnen daran, sich mit anderen Gruppen stärker zu vernetzen und an für die Bewegung relevanten Orten, wie etwa dem Sitz des EU-Parlamentes und der EUKommission, zu demonstrieren. Im März 2013 findet die Refugees' Revolution

6 MUJAOist eine Abspaltung von Al Quaida im Maghreb und fokussiert sich auf Westafrika. 
Bustour statt - als Versuch, wieder politisch in die Offensive zu kommen und die Fragen der Ressourcen und der sich daraus ergebenden Konflikte zurück treten zu lassen. ${ }^{7}$ Sie dient den AktivistInnen vor allem dazu, Asylbewerberunterkünfte in Deutschland aufzusuchen und über die Bewegung zu informieren. Zur Ankunft der Bustour wird in München der Non-Citizen-Kongress veranstaltet, u.a. organisiert von den InitiatorInnen der Refugee Tent Action, auf dem erstmals eine Theoriebildung aus der Bewegung heraus unternommen wird. ${ }^{8}$

Die Besetzung des Oranienplatzes erzeugt zwar wesentlich weniger Öffentlichkeit und wird mit weitaus weniger Repression von staatlicher Seite beantwortet als der Hungerstreik vor dem Brandenburger Tor zur Weihnachtszeit, doch 2013 - mit zunehmender Präsenz der Refugee-Bewegung in der bundesdeutschen Öffentlichkeit - steigt der Druck auf die BesetzerInnen. Während der NSU-Prozess ab Mai 2013 startet und Einblicke in ein neonazistisches Terrornetzwerk offenbart, beginnen Neonaziströmungen in Deutschland die Refugee-Bewegung als Angriffsziel auszumachen. Zudem haben mit den Krisenprotesten auch reaktionäre und neonazistische soziale Bewegungen Zulauf bekommen. Die Zahl registrierter Angriffe auf AsylbewerberInnenunterkünfte verdoppelt sich zwischen 2012 und 2013 (vgl. Peters: 2014). Ähnlich wie Anfang der 1990er Jahre greifen auch rechte und konservative PolitikerInnen das Thema auf. Berlins Innensenator Frank Henkel (CDU) fordert 2013 den Bezirk auf, den Platz zu räumen.

Innerhalb der Refugee-Bewegung ist die Bedeutung des Platzes für die öffentliche Präsenz der Bewegung unumstritten. Der Druck auf die BesetzerInnen, einer Verhandlungslösung zuzustimmen, steigt jedoch Anfang 2014 erheblich, als SPD-Senatorin Dilek Kolat ein Ergebnis herbeiführen soll, das die einzige Alternative zur Räumung darstelle. Die AktivistInnen sind gespalten, sehen in der Neubewertung von 300 bis 500 Asylverfahren - hier variieren die offiziellen Zahlen - ein Scheinangebot und behalten Recht, als zwei Wochen nach der Räumung des Platzes die ersten Abschiebungen Geflüchteten drohen, die in die Verhandlungen einbezogen waren. Zwei Monate nach der Räumung des Oranienplatzes soll die Gerhard-Hauptmann-Schule im Juni 2014 schließen. Eine Gruppe von etwa 40 Geflüchteten, MigrantInnen und UnterstützerInnen widersetzt sich der Räumung. Als ein Großaufgebot an Polizeikräften aus ganz Deutschland gewaltsam räumen will, drohen einige wenige Geflüchtete vom Dach zu springen. Zehn Tage lang sind bis zu drei Häuserblocks um die Schule Sperrgebiet und tausende Polizisten aus dem ganzen Land rund um die Uhr im Einsatz. Trotz des Ausschlusses der Presse ist das Thema nach spätestens einer

7 Blog der Refugees Revolution Bustour: refugeesrevolution.blogsport.de.

8 Blog der Non-Citizen Gruppe: refugeestruggle.org. 
Woche in den Mainstreammedien präsent. Zur selben Zeit ist der Freedom-notFrontex-Marsch in Brüssel angekommen, um beim Treffen des Europäischen Rates gegen die Flüchtlingspolitik der EU zu demonstrieren. Die Bewegung hat sich inzwischen auch auf EU-Ebene vernetzt.

Das Bewusstsein, den Sprung vom Protest zur Bewegung vollzogen zu haben, drückt sich auch in dem neuen Slogan „You can't evict a movement“ aus, der bei den Protesten gegen die Gerhard-Hauptmann-Schule geprägt wird. Die Auseinandersetzungen um die Gerhard-Hauptmann-Schule und weitere Räumungen führen erstmals seit Beginn der Bewegung zu einer Vernetzung von den ebenfalls in Berlin und Hamburg gegenwärtig starken Recht-auf-die-Stadt-Bewegung mit der Refugee-Bewegung - wie etwa im United-Neighbours-Bündnis. ${ }^{9}$ Hier zeigt sich, dass eine Überwindung der „stark fragmentierte(n) Strukturen der Subalternität“, die die „Konflikte in der neoliberalen Stadt“ (Holm 2014: 23) hervor gebracht haben, nicht automatisch in eine Zunahme von Rassismen, Ausgrenzung und Vertreibung münden. Die bereits 2013 von der Gruppe „Lampedusa in Hamburg" formulierte Forderung nach Aufenthalt nach Paragraf 23 Aufenthaltsgesetz (AufenthG) wird nach einigen Tagen zu einer bundesweiten Kampagne, der sich vor allem auch ProtagonistInnen des Kulturbetriebs und der Universitäten in Deutschland anschließen. ${ }^{10}$ Doch weder in Hamburg noch in Berlin wird von diesem Mittel Gebrauch gemacht. Den ganzen Sommer über besetzen in Berlin AktivistInnen der Refugee-Bewegung verschiedene Orte in der Stadt - wie u.a. den Fernsehturm, den Checkpoint Charlie, die Gedächtniskirche oder das DGB-Gebäude. Alle Gebäude werden entweder sofort oder nach Verhandlungen geräumt, Ergebnisse im Sinne der Bewegung gibt es nicht. Stattdessen wird der Druck auf die AktivistInnen durch die Räumung der im Zuge der Verhandlungen bereitgestellten Unterkünfte, verstärkte Polizeikontrollen auf Straßen und öffentlichen Plätzen und Verhaftungen weiter verstärkt.

\section{Die gesamtgesellschaftliche Bedeutung der Refugee-Proteste}

Wieso wecken die Proteste eine so martialische Abwehr, wenn es doch möglich wäre, mit Instrumenten wie der Gewährung von Aufenthalt nach Paragraf 23 eine Befriedung zu erreichen? Es zeigt sich, dass es um weit mehr geht als um den Aufenthalt nach Paragraf 23. Es geht auch um die im Zuge der Krise offen agierende EU-Kern-Macht Deutschland und die Rollenteilung der EU in

9 Vgl.asylstrikeberlin.wordpress.com/2014/09/15/27-9-united-neighbours-demonstration.

10 Unter anderem siehe „Yalla $\$ 23$ “ unter https://www.facebook.com/pages/ Yallah-23/1497017647179932 
„Ränder" und „Krisen-Staaten“ auf der einen und ein zunehmend hegemoniales „Kern-Europa“ auf der anderen Seite, das sich aufgrund der Dublin-Regelungen nicht mit dem Geschehen an seinen „Rändern“ befassen muss. Darüber hinaus geht es um die Umsetzung der Krisenprogramme oder auch eine erneute neoliberale Restrukturierung der staatlichen Strukturen. Der allgemeine Abbau des Wohlfahrtsstaates wird von umfassenden "Kontroll- und Zwangsmaßnahmen“ und gesellschaftlichen Spaltungen und Ausgrenzungen begleitet (Butterwege 2013: 16ff.), von denen MigrantInnen und Geflüchtete wiederum am stärksten betroffen sind. Sie sind das Experimentierfeld für die Durchsetzung dieser Politik, an der (ausbleibenden) Empörung und Solidarisierung mit ihnen lässt sich ablesen, was durchsetzbar ist - diskursiv und praktisch.

Die Asyl- und Migrationspolitik demonstriert nach dem Verlust der Möglichkeit, Zustimmung durch die Verteilung von Profiten und materielle Konzessionen zu generieren, die „Handlungs- und Durchsetzungsfähigkeit“ des Nationalstaates (vgl. Schwenken/Benz 2005: 367). Insofern sind - ähnlich wie Anfang der 1990er Jahre - rassistische Diskurse und Attacken bis zu einem gewissen Maß opportun für die Zurückweisung einer Verantwortung Deutschlands für das Sterben auf dem Mittelmeer und die Wiedereinführung von Grenzkontrollen im Schengenraum Herbst 2014. Solange der Zwang und die Kontrolle als notwendige Schutzmaßnahme dargestellt werden können und nicht in Relation zu den seit letztem Jahr bekannten Überwachungen durch in- und ausländische Geheimdienste gesetzt werden, solange der Diskurs von den, illegitimen' Grenzen wie der Berliner Mauer und den ,legitimen' Grenzen des Schengenraums aufrechterhalten werden kann, stehen eine gesamtgesellschaftliche Solidarisierung gegen Austeritätsprogramme und eine transnationale Solidarisierung gegen die Rollenverteilung in der EU und eine neokoloniale Außenpolitik der EU nicht in Aussicht. Eine „allgemeine gesellschaftliche Angst“, „die Furcht vor gefährlichen Fremden und unbekannten Bedrohungen" generiert weiterhin Akzeptanz (vgl. Hardt/Negri 2013: 30f.), doch Solidarisierungen wie die Zusammenarbeit von Recht-auf-die-Stadt- und Refugee-Bewegung zeigen auch, dass Ängste und (gegenseitige) Ressentiments partiell überwunden werden können.

Mit ihrem Protest haben die MigrantInnen und Geflüchteten einen Rollenwechsel vollzogen: von Verhandlungsmasse und Projektionsfläche in diesen Auseinandersetzungen zwischen den Ländern im Osten oder Süden der EU und den nördlichen Staaten zu einem unkalkulierbaren Risiko für die Fortsetzung der FRONTEX-Grenzpolitik unter der Ägide des Nordens. Hier bestätigt sich die Theorie von der „Autonomie der Migration“ im Sinne der Interpretation von Sandro Mezzadra als einem widerständigen und konfliktiven Prozess von Subjekten, deren Subjekt-Werden schon Teil des Widerstands ist. Der mit den verschiedenen Wellen neoliberaler Anpassung etablierte gesellschaftliche Modus 
Operandi der Diskriminierung, Entsolidarisierung und Ausgrenzung gerät ins Stocken.

Die „Schengener Grenze“ als „undemokratischste Institution Europas“(Tsianos 2014: 7) ist ein Synonym dafür, wie weit dieses Kontroll- und Ausgrenzungsregime trotz zehntausenden Toten im Mittelmeer und an den Grenzzäunen Europas akzeptiert wird. Es ist die Barbarei, die an den „Rändern“ stattfindet und die durch die Externalisierung der Außengrenzen der EU und ihre Verschiebung nach Nordafrika zunächst bis zur Krise und dem Arabischen Frühling nicht bis nach „Zentral-Europa“ vordrang. Nun sind mit den Geflüchteten des LibyenKrieges, der Mali-Intervention und des Syrienkrieges, mit der Zuspitzung von Ausgrenzung und Rassismus im Zuge der Krise und dem weiteren Abbau von sozialstaatlichen Leistungen und Subsistenzmöglichkeiten diejenigen, die vor diesen Kriegen und Krisen fliehen, plötzlich präsent in Deutschland und fordern die Achtung ihrer Menschenrechte ein.

\section{Die Bedeutung der Refugee-Proteste für das neue Europa in der Krise}

Die Krise in Europa, die Kasparek und Tsianos auch als Krise Schengens und Indiz für ein "grundlegende(s) Ungleichgewicht in der territorialen Konstruktion der EU“ beschreiben (Kasparek/Tsianos 2013: 71), geht einher mit erneuter nationalistischer Abgrenzung. Das Nord-Süd-Gefälle in der EU, das in der Krise offensichtlich geworden ist, wird im Zuge einer erneuten neoliberalen Restrukturierung als selbst verschuldet propagiert. Dies verschleiert auch, dass die Schaffung des sogenannten Schengenraums und der „Drittstaatenregelungen“ eine EU-weit koordinierte Migrationspolitik nach den Interessen des sich stetig herausbildenden „Kern-Europas“ bedeutet (vgl. Nuscheler 2011: 301ff.).

Die 2004 gegründete Grenzschutzagentur FRONTEX fungiert, so Kasparek (2013), als „die Verfestigung eines Prozesses, mittels dessen eine europäische Außengrenze überhaupt erst als politische Einrichtung entsteht“. So wird eine „Nicht-Bevölkerung“" geschaffen, die dauerhaft „auf der Grenze“ (Hermann 2014: 163) lebt. Die Gruppe Transit Migration (2005: 359) stellte fest, dass die „Extensionspolitik der Migrationskontrollen dazu bei[trägt], die Ränder der Europäischen Union zu Hotspots des Migrationsgeschehens zu machen“. Die Dublin-Regelungen schreiben diese Rollenzuweisung unter den EU-Staaten fest. Der Schengenraum, die Errichtung eines FRONTEX-Grenzregimes und die fortwährende Einschränkung der Bewegungsfreiheit gehen für Geflüchtete und MigrantInnen in der Regel mit einem zunehmenden Mangel an Zugängen zu den eigenen Ressourcen einher, notwendigen Dienstleistungen und Waren, zu 
Informationen und Bildung - oftmals auch mit der Zunahme von kriegerischen Konflikten. Die neoliberale Globalisierung bewirkt hier durch Freihandelsabkommen, den Ausverkauf von Ressourcen und Land in einem immer schnelleren und größeren Maßstab und den damit einhergehenden Vertreibungen und Verwüstungen eine stetige Erhöhung des Migrationsdrucks.

\section{Im Zyklus der Krisenproteste und des Arabischen Frühlings: die transnationale Dimension der Refugee-Proteste}

Wenn 2011 „ein Jahr der Proteste, Revolutionen und des politischen Wechsels“ war, ein „tipping point, where discontent turned into worldwide protests that spread and amplified each other" (Fuchs 2014: 17), dann stellt sich die Frage, was dazu geführt hat, dass aus einer Handvoll AsylbewerberInnen, die nach dem Suizid eines Genossen in den Hungerstreik treten, die erste Refugee-Bewegung in Deutschland entstehen konnte und ob es möglicherweise einen Zusammenhang zwischen den weltweiten Protesten gibt, insbesondere mit dem Arabischen Frühling, der am Anfang dieses Protestzyklus steht. Es gibt einen doppelten Zusammenhang: Einerseits trieben die dem Arabischen Frühling nachfolgenden bewaffneten Konflikte und Militärinterventionen von Libyen über Malibis nach Syrien immer mehr Menschen in die Migration und zur Flucht. Zudem waren die Flucht- und Migrationsrouten nicht mehr so gut kontrollierbar und die neu gebildeten Regierungen nicht immer so willige Erfüllungsgehilfen der EU wie die alten. Andererseits scheint der Arabische Frühling auch neben dem realen einen imaginativen Möglichkeitsspielraum eröffnet zu haben, in dem Demokratie und das Recht auf Kommunikation nicht mehr nur utopisch erschienen. Diese Forderungen werden in den Bewegungen des Protestzyklus vom Arabischen Frühling über Occupy und M15 bis zum Movimento Passa Livre und dem Gezi-Park-Besetzung zum Ziel und zur revolutionären Praxis. Sie entwickeln die Organisationsformen und Kommunikationsformen globalisierungskritischer Bewegungen des vorlaufenden Protestzyklus weiter.

Zur Quelle der Inspiration waren Mitte der 1990er Jahre - nach dem Scheitern autoritärer antikapitalistischer Gruppen und Systeme -die ZapatistInnen geworden, und zwar wegen ihrer Betonung des transformatorischen Prozesses und ihrer Absage an eine bloße Übernahme staatlicher Macht. Die horizontale Organisation des Weltsozialforums und vieler globalisierungskritischer Bewegungen, die Hervorhebung des Prozesses ist bereits in der Auseinandersetzung mit der Geschichte realsozialistischer und kommunistischer Politik entstanden - nun gingen die Bewegungen in dem hier betrachteten Protestzyklus einen Schritt weiter: Sie verbinden die Versammlung auf öffentlichen Plätzen und eine 
internetgestützte Kommunikation mit den Forderungen nach Basisdemokratie und Transparenz - Manuel Castells bezeichnet sie daher als „networked social movements" (vgl. Castells: 2012).

Das Camp am Oranienplatz ist analog zu den Besetzungen von öffentlichen Plätzen von Kairo und New York bis Tel Aviv und Istanbul das Mittel der sozialen Bewegungen dieses Protestzyklus, an die Öffentlichkeit zu treten. Die Besetzung des fortwährend privatisierten öffentlichen Raumes und das Durchbrechen von Kommunikationssperren, im Fall der Refugee-Bewegung weniger gegeben durch die Sperrung widerständiger Blogs und Social-Web-Applikationen als vielmehr durch den Bruch der Isolation in Lagern und durch die Residenzpflicht sowie das Arbeits- und Studienverbot, ermöglicht die öffentliche Präsenz und die Kommunikation mit einer breiteren Öffentlichkeit. „The prison camp, the protest camp and the refugee camp are three key spaces through which contemporary power struggles between states and peoples are being articulated“, schreibt Ramadan (2013: 146) über die Besetzung des Tahrir-Platzes. Die Besetzung öffentlicher Räume und ihre Rückgewinnung gegen die Überwachung und den Zwang und für gelebte Demokratie und Kommunikation scheinen symptomatisch für sämtliche soziale Bewegungen dieses Protestzyklus zu sein und setzen den Mechanismen der Ausgrenzung und Kontrolle, die mit der Krise weiter ausgebaut wurden, Transparenz, Öffentlichkeit und Formen der Partizipation entgegen.

\section{Ausblick}

Die Refugee-Proteste haben sich in Deutschland ab 2012 erstmals zu einer sozialen Bewegung entwickelt. Die Protestwellen, wie es sie seit 1945 immer wieder gab, und die vor allem Mitte der 1990er Jahre zu größeren Mobilisierungen führten, haben ab 2012 eine Vernetzung von MigrantInnen und Geflüchteten bewirkt, die über den nationalen Rahmen hinaus geht und auch in der breiteren Öffentlichkeit präsent ist. Die vom Asylstrike bzw. Refugee March Mitte 2012 erhobenen Forderungen richten sich auf eine grundsätzliche Teilhabe und die Aufhebung des Antagonismus von Citizen und Non-Citizen, so Refugee Tent Action (2012) und gehen daher über partikulare Interessen weit hinaus. Sie greifen damit auch das gesamtgesellschaftliche Problem von Ausgrenzung, Diskriminierung und Prekarisierung auf, was sich durch verschiedene Wellen neoliberaler Anpassung verstärkt hat. Die Forderung einer gleichberechtigten Teilhabe hat in Zeiten der Krise und in „neoliberalisierenden“Städten eine erhebliche Sprengkraft. Ähnlich wie zu Beginn der 1990er Jahre sind bisher trotz anhaltender Proteste daher kaum Erfolge auf legislativer Ebene zu verzeichnen. Vielmehr ist der Repressionsdruck erheblich verstärkt worden und zunehmend beherrschen rassistische Stereotype 
die öffentlichen Diskurse. Neonazistische und rassistische Gegenbewegungen sind ebenfalls gewachsen.

Doch die Stärke der Refugee-Bewegung liegt auch darin, dass sie gesamtgesellschaftliche Entwicklungen anspricht und ihre Auswirkungen - sowohl jenseits wie diesseits der Grenzen Europas. Die Antwort ist auch der Slogan „Wir sind hier, weil ihr unsere Länder zerstört“. Wenn sich Proteste und Bewegungen auf die Durchsetzung des Bleiberechts sowie die Kritik neokolonialer und postfordistischer Akkumulationsregimes fokussieren und sich die Verbindung zwischen Migrantenprotesten und Global Justice Movements festigt, dann wird das ganze Potenzial der Bewegung sichtbar. Aus transnationaler Perspektive sind die Refugee- und MigrantInnen-Proteste und ihre Bewegungen auch ihrer Zielsetzung und Thematik nach Teil des aktuellen Protestzyklus.

Die gegenwärtig zu beobachtende weltweite Zunahme an Kriegen und militärischen Konflikten und die Bereitschaft zu martialischen (Re-)Aktionen ist auch der Kultur von Zwang, Kontrolle und Ausgrenzung geschuldet und dem Mangel an konstruktiven Perspektiven. Der Protestzyklus von 2011, in dem sich die Refugee-Proteste verorten, weist in die andere Richtung, doch nur die Entwicklung einer starken Global Justice Movements mit Refugee-Bewegungen kann eine gegen-hegemoniale Kraft befördern.

\section{Literatur}

Arps, Jan Ole (2014): Engagiert statt gleichgültig. Die Proteste rund um die besetzte Schule in Berlin-Kreuzberg werden von vielen Menschen unterstützt. In: neues deutschland, 9.7.2014.

Benz, Martina/Schwenken, Helen (2005): Jenseits von Autonomie und Kontrolle: Migration als eigensinnige Praxis. In: PROKLA 140 35(3): 363-377.

Bouali, Kerima/Gude, Sigmar (2014): Gentrifizierung oder Wiederkehr der Wohnungsnot? In: Holm, Andrej (Hg.): Reclaim Berlin. Soziale Kämpfe in derneoliberalen Stadt. Berlin-Hamburg: 27-49.

Brand, Ulrich (2012): Contradictions and crises of neoliberal-imperial globalization and the political opportunity structures for the Global Justice Movements. In: Innovation - the European Journal of Social Science Research 25(3): 283-298.

Butterwege, Christoph (2013): Marktradikalismus und Rechtspopulismus. Neoliberale Modernisierung als Türöffnerin für rassistische Ausgrenzung und Standortnationalismus. In: Friedrich, Sebastian/Schreiner, Patrick (Hg.): Nation, Ausgrenzung, Krise. Kritische Perspektiven auf Europa. Münster: 14-28.

Castells, Manuel (2012): Networks of Outrage and Hope. Social Movements in the Internet Age. Cambridge.

Fuchs, Christian (2014): Occupy Media! The occupy movement and social media in crisis capitalism. London.

Hardt, Michael/Negri, Antonio (2013): Wofür wir kämpfen. Frankfurt/M.

-/Negri, Antonio (2003): Empire. Die neue Weltordnung. Frankfurt/M.

Herrmann, Goetz (2014): Ein Mehr an Freiheit durch ein Mehr an Sicherheit? Zur Organisation von Zirkulation in der Europäischen Union: Die Beispiele des Grenz- und Migrationsmanagements. 
In: Andreas Vasilache (Hg.): Gouvernementalität, Staat und Weltgesellschaft, Staat-Souveränität-Nation. Wiesbaden: 137-168.

Holm, Andrej (2014): Reclaim Berlin. In: Andrej Holm, (Hg.): Reclaim Berlin. Soziale Kämpfe in der neoliberalen Stadt. Berlin-Hamburg: 7-24.

Josten, Daniel (2012): „Die Grenzen kann man sowieso nicht schließen“. Migrantische Selbstorganisation - zivilgesellschaftliches Engagement zwischen Ausschluss und Partizipation. Münster.

Kasparek, Bernd/Tsianos, Vassilis S. (2013): Out of control? Schengen und die Krise. In: Friedrich, Sebastian/Schreiner, Patrick (Hg.): Nation, Ausgrenzung, Krise. Kritische Perspektiven auf Europa. Münster: 65-78.

- (2013): Von Schengen nach Lampedusa, Ceuta und Piräus: Grenzpolitiken der Europäischen Union. URL: http://www.bpb.de/apuz/172382/von-schengen-nach-lampedusa-ceuta-undpiraeus-grenzpolitiken-der-europaeischen-union?p=all. Zugriff: 20.8.2014.

Koopmans, Ruud (2004): Protest in Time and Space: The Evolution of Waves of Contention. In: Snow, David A./Soule, Sarah A./Kriesi, Hanspeter (Hg.): The Blackwell Companion to Social Movements. Malden-Oxford-Carlton: 19-46.

Lebuhn, Henrik (2013): Migration - Recht - Citizenship. Potentiale und Grenzen eines kritischen Diskurses. In: Paul Mecheril et al. (Hg.): Migrationsforschung als Kritik? Spielräume kritischer Migrationsforschung. Wiesbaden: 231-244.

Mayer, Margit (2013a): Contradictions in urban activism in the context of neoliberalization. Rede auf der Konferenz: Rethinking Urban Social Movements, Gothenburg 22.5.2013. URL: http://www.socav.gu.se/digitalAssets/1436/1436845_rethinking-urban-movements-program. pdf. Zugriff: 26.8.2014.

- (2013b): Urbane soziale Bewegungen in der neoliberalisierenden Stadt. In: sub/urban - Zeitschrift für kritische Stadtforschung 1(1): 155-168.

Mezzadra, Sandro (2011): The Gaze of Autonomy, Capitalism, Migration and Social Struggles. In: Squire V.London: URL: http://mcrg.ac.in/Development/draft_Symposium/Sandro.pdf. Zugriff: 17.8.2014.

- (2010): Autonomie der Migration - Kritik und Ausblick. Eine Zwischenbilanz. In: Grundrisse. Zeitschrift für linke Theorie \& Debatte, Nr. 34: 22-29.

Moulier Boutang, Yann (2002): Nicht länger Reservearmee. In: Jungle World Nr. 15, 3.4.2002.

Neidhardt, Friedhelm (Hg.) (1994): Öffentlichkeit, öffentliche Meinung, soziale Bewegungen. Opladen:.

Nuscheler, Franz (2011): Migrationsgovernance in der EU. In: Georg Simonis/Helmut Elbers (Hg.): Externe EU-Governance. Wiesbaden: 299-314.

Omwenyeke, Sunny (2004): Interview mit der „Karawane für die Rechte der Flüchtlinge und MigrantInnen“. In: Alabi, Ade et al.: Keine Romantisierung, bitte. „Autonomie der Migration im Streitgespräch". URL: http://thecaravan.org/node/19. Zugriff: 24.8.2014.

Papadopoulos, Dimitris et al. (2008): Espace Routes. Control and Subversion in the $21^{\text {st }}$ century. London-Ann Arbor.

Peters, Anna (2014): Angriffe auf Asylbewerberheime nehmen zu. URL: http://www.dw.de/ angriffe-auf-asylbewerberheime-nehmen-zu/a-17476821. Zugriff: 5.11.2014.

Plöger, Andrea (2014): Eine andere Welt sichtbar machen. Mediale Repräsentation, Strategien und Kommunikation im Weltsozialforumsprozess. Dissertation. URL: http://www.diss.fu-berlin. de/diss/receive/FUDISS_thesis_000000096295.Zugriff: 22.10.2014.

Ramadan, Adam (2013): From Tahrir to the world: The camp as a political public space. In: European, Urban and Regional Studies 20(1): 145-149.

Refugee Tent Action (2012): Warum leisten wir Widerstand. Pressemitteilung, 31.8.2012. URL: http://www.refugeetentaction.net. Zugriff: 25.8.2014. 
Rucht, Dieter/Heitmeyer, Wilhelm (2008): Mobilisierung von und für Migranten. In: Roth, Roland/Rucht, Dieter (Hg.): Die Sozialen Bewegungen in Deutschland seit 1945. Ein Handbuch. Frankfurt/M: 573-592.

Schwenken, Helen (2007): Schwierige Allianzen: Lobbypolitik aus der Perspektive „schwacher AkteurInnen". In: PERIPHERIE 105/106: 35-56.

Tarrow, Sidney (1998): Power in Movement. Social Movements and Contentious Politics. Cambridge. Tilly, Charles (2004): Social Movements, 1768-2004. Boulder-London.

Transit Migration (2005): Turbulente Ränder. Konturen eines neuen Migrationsregimes im Südosten Europas. In: PROKLA 140 35(3): 345-362.

Tsianos, Vassilis S. (2014): Jenseits der Staatsbürgerschaft. In: die tageszeitung, 24/25.5.2014: 7. Wallerstein, Immanuel (1984): Der historische Kapitalismus. Berlin.

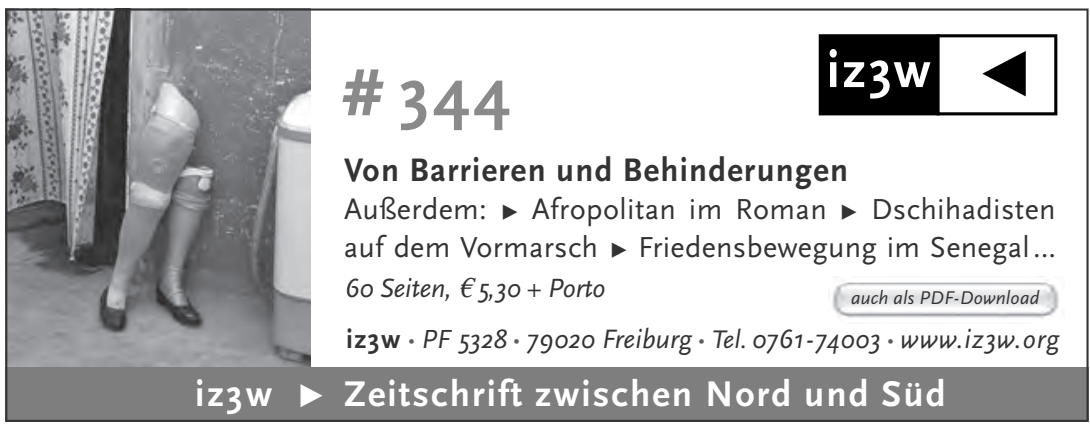

